\title{
Neuroprotection on Multiple Sclerosis: A BDNF Perspective
}

\section{Junhua Xiao ${ }^{1,2,3 *}$}

${ }^{1}$ Centre for Neuroscience Research, The University of Melbourne, Victoria 3010, Australia

${ }^{2}$ Department of Anatomy and Neuroscience, The University of Melbourne, Victoria 3010, Australia

${ }^{3}$ Florey Neuroscience Institutes, The University of Melbourne, Victoria 3010, Australia

Multiple sclerosis (MS) is a progressive, chronic central nervous system (CNS) inflammatory demyelinating disease. It is the most common neurological disease in middle-aged adults, striking three times more women than men with diagnosis peaking at around 30 years of age, thus affecting people in their most productive years. It has a lifelong impact and its prevalence is increasing steadily over time. Investment in research that would delay or ideally prevent the progression could bring substantial rewards in terms of both reducing the financial burden and increasing quality of life.

The disease is thought to result from an inflammatory attack against myelin, which is produced by specialised glial cells in the CNS known as oligodendrocytes, and their death are commonly observed elements in MS lesions [1]. As a result, the efficient transmission of neuronal signals is disrupted, causing nervous system dysfunction. Once the disease presents, the condition is permanent and degenerative. An endogenous repair process often follows the death of these glial cells, which is effected by surviving oligodendrocytes in the lesion area and complemented by the recruitment of oligodendrocyte precursor cells (OPCs). This repair process is variable, but can result in the return to relatively normal CNS function. However over time and following successive demyelinating events, the myelin repair is ultimately insufficient, invariably leading to irreversible axonal damageand progressive and extensive disability $[2,3]$. Currently the failure of remyelination remains a major obstacle to recovery in MS patients and strategies aimed at improving and enhancing remyelination is critically important to complement the currently available immunomodulatory treatments. In this regard, studying molecules involved in oligodendrocyte myelination has the potential to identify novel therapeutic approaches and strategies to promote remyelination. Recently, increasing evidence suggest that Brain-Derived Neurotrophic Factor (BDNF) not only enhances CNS myelination during development [4-6], but also exerts neuroprotective roles following demyelination in animal models of MS [7,8].

BDNF is a member of the neurotrophin family of growth factors, and signals through two distinct classes of transmembrane receptors: the tropomyosin-related kinase $\mathrm{B}$ receptor $(\operatorname{TrkB})$ and the structurally unrelated p75 neurotrophin receptor (p75NTR) $[9,10]$. Previous studies have shown that BDNF plays a key role in regulating CNS myelination, as evidenced by myelin deficits in both BDNF knockout as well as heterozygous mice [4-6]. In vitro studies have also suggested that BDNF can exert a number of effects upon the oligodendroglial lineage including proliferation, differentiation, maturation and myelination $[6,11,12]$. Recent studies have implicated that BDNF exerts functionally neuroprotective roles following CNS demyelination in animal models of demyelinating diseases including experimental autoimmune encephalomyelitis (EAE) and toxic models of demyelination such as cuprizone. In response to the cuprizone model of demyelination, BDNF heterozygote mice exhibit greater demyelination and reduced remyelination in the absence of change to the number of oligodendrocytes, microglia and astrocytes as well as the extent of axonal injury [8]. A more severe course of EAE and increased axonal loss was observed in mice in which BDNF has been selectively deleted in GFAP positive cells such as oligodendrocytes, astrocytes and some neurons [7]. And it is more effective in reducing clinical severity and structural damage when the BDNF level is modulated at initial stages of EAE versus later stages [13]. Thus, this implicates there may be an early window of therapeutic opportunity for the modulation of BDNF levels, at least in EAE.

In the CNS, BDNF can be derived from both CNS resident cells and immune cells. In the normal CNS, BDNF is primarily derived from neurons [14]. However, following demyelination such as in MS and EAE, activated astrocytes express increased levels of endogenous BDNF $[7,15,16]$. In addition, BDNF can be produced by subtypes of immunecells such as activated Tcells, B-cells and monocytes $[15,17]$. Thus, BDNF is well placed to exert a modulatory effect upon myelination in this context $[15,18]$. However, CNS-derived BDNF appears to play a critical neuroprotective role in autoimmune demyelination. Mice deficient for BDNF in T-cells exhibited progressive disability and enhanced axonal loss in EAE and mice overexpressing BDNF in T-cells exhibited less severe EAE and axonal protection [7]. However, experiments utilising bone marrow chimeras reveal that immune cell-derived BDNF cannot influence disease severity following CNS deletion of BDNF [13]. This suggests that CNS resident cells are the major source of biologically relevant $\mathrm{BDNF}$ in autoimmune demyelination. Whether in this context BDNF exerts its influence directly on the myelinated axon / neuronoligodendrocyte interface remains an open question.

The next would be to identify the molecular targets of BDNF in autoimmune demyelination. As the CNS myelination is normal in p75NTR knockout mice [6], BDNF receptor TrkB appears to be the key player in regulating the promyelinating effect of BDNF. In vitro studies have identified that BDNF exerts several influences upon oligodendroglial lineage such as proliferation, differentiation, maturation and myelination by activating oligodendroglial-expressed TrkB receptors $[6,11,12]$. In addition to oligodendroglial lineage cells, TrkB receptors are also present in neurons and astrocytes as well as infiltrating immune cells in the CNS $[18,19]$. Thus in this context, there is no shortage of potential BDNF targets. Interestingly, the activity of TrkB was increased in the neurons of EAE-diseased mice

*Corresponding author: Junhua Xiao, Centre for Neuroscience Research, The University of Melbourne Victoria 3010, Australia, Tel: (+61 3) 8344 7572; Fax: (+61 3) 9349 4432; E-mail: xiaoj@unimelb.edu.au

Received May 14, 2012; Accepted May 14, 2012; Published May 17, 2012

Citation: Xiao J (2012) Neuroprotection on Multiple Sclerosis: ABDNF Perspective J Neurol Neurophysiol 3:e108. doi:10.4172/2155-9562.1000e108

Copyright: (c) 2012 Xiao J. This is an open-access article distributed under the terms of the Creative Commons Attribution License, which permits unrestricted use, distribution, and reproduction in any medium, provided the original author and source are credited. 
compared to the intact axons of control mice, suggesting BDNF is at least exerting a neuronal effect in this context [7]. In addition to significantly delay EAE onset and reduce clinical severity, treatment with BDNF engineered bone marrow stem cells also reduced the expression of pro-inflammatory cytokines and increased expression of anti-inflammatory cytokines [20]. Furthermore, immune cell expression of TrkB has been show to play an important role in MS immunopathogenesis by modulating auto-reactive $\mathrm{T}$ cell survival and behaviour [18]. Collectively, these implicate that TrkB could be a key target of BDNF's neuroprotective role in autoimmune demyelinating diseases such as MS.

What is beyond dispute however is that regardless of the model or the strategy to manipulate BDNF expression, the effect it exerts is a uniformly promyelinating one. Given the difficulties inherent with using the recombinant neurotrophins themselves to treat demyelinating and other CNS disorders, increasing attention has turned to the development of alternative strategies to harness neurotrophic action for clinical use. One means of doing this is to use functional mimetics of neurotrophins. In the context of BDNF, the compounds that have the advantage of being able to selectively target TrkB receptors with appropriate pharmacokinetic properties could have a strong therapeutic potential for treating human demyelinating diseases such as MS in the future.

\section{References}

1. Barnett MH, Prineas JW (2004) Relapsing and remitting multiple sclerosis: pathology of the newly forming lesion. Ann Neurol 55: 458-468.

2. Kilpatrick TJ, Butzkueven H, Grigg A (2002) Prospects for stem cell transplantation in multiple sclerosis. J Clin Neurosci 9: 361-367.

3. Franklin RJ, Ffrench-Constant C (2008) Remyelination in the CNS: from biology to therapy. Nat Rev Neurosci 9: 839-855.

4. Cellerino A, Carroll P, Thoenen H, Barde YA (1997) Reduced size of retinal ganglion cell axons and hypomyelination in mice lacking brain-derived neurotrophic factor. Mol Cell Neurosci 9: 397-408.

5. Vondran MW, Clinton-Luke P, Honeywell JZ, Dreyfus CF (2010) BDNF+/- mice exhibit deficits in oligodendrocyte lineage cells of the basal forebrain. Glia 58 : 848-856.

6. Xiao J, Wong AW, Willingham MM, van den Buuse M, Kilpatrick TJ, et al. (2010) Brain-Derived Neurotrophic Factor Promotes Central Nervous System Myelination via a Direct Effect upon Oligodendrocytes. Neurosignals 18: 186202.
7. Linker RA, Lee DH, Demir S, Wiese S, Kruse N, et al. (2010) Functional role of brain-derived neurotrophic factor in neuroprotective autoimmunity: therapeutic implications in a model of multiple sclerosis. Brain 133: 2248-2263.

8. VonDran MW, Singh H, Honeywell JZ, Dreyfus CF (2011) Levels of BDNF impact oligodendrocyte lineage cells following a cuprizone lesion. J Neurosci 31: $14182-14190$.

9. Huang EJ, Reichardt LF (2001) Neurotrophins: roles in neuronal development and function. Annu Rev Neurosci 24: 677-736.

10. Chao MV (2003) Neurotrophins and their receptors: a convergence point for many signaling pathways. Nat Rev Neurosci 4: 299-309.

11. Van't Veer A, Du Y, Fischer TZ, Boetig DR, Wood MR, et al. (2009) Brainderived neurotrophic factor effects on oligodendrocyte progenitors of the basa forebrain are mediated through trkB and the MAP kinase pathway. J Neurosci Res 87: 69-78.

12. Du Y, Lercher LD, Zhou R, Dreyfus CF (2006) Mitogen-activated protein kinase pathway mediates effects of brain-derived neurotrophic factor on differentiation of basal forebrain oligodendrocytes. J Neurosci Res 84: 1692-1702.

13. Lee DH, Geyer E, Flach AC, Jung K, Gold R, et al. (2011) Central nervous system rather than immune cell-derived BDNF mediates axonal protective effects early in autoimmune demyelination. Acta Neuropathol 123: 247-258.

14. Lewin GR, Barde YA (1996) Physiology of the neurotrophins. Annu Rev Neurosci 19: 289-317.

15. Stadelmann C, Kerschensteiner M, Misgeld T, Brück W, Hohlfeld R, et al. (2002) BDNF and gp145trkB in multiple sclerosis brain lesions: neuroprotective interactions between immune and neuronal cells? Brain 125: 75-85

16. Burbach GJ, Hellweg R, Haas CA, Del Turco D, Deicke U, et al. (2004) Induction of brain-derived neurotrophic factor in plaque-associated glial cells of aged APP23 transgenic mice. J Neurosci 24: 2421-2430.

17. Kerschensteiner M, Gallmeier E, Behrens L, Leal VV, Misgeld T, et al. (1999) Activated human $\mathrm{T}$ cells, B cells, and monocytes produce brainderived neurotrophic factor in vitro and in inflammatory brain lesions: a neuroprotective role of inflammation? J Exp Med 189: 865-870

18. De Santi L, Annunziata P, Sessa E, Bramanti P (2009) Brain-derived neurotrophic factor and TrkB receptor in experimental autoimmune encephalomyelitis and multiple sclerosis. J Neurol Sci 287: 17-26.

19. Cahoy JD, Emery B, Kaushal A, Foo LC, Zamanian JL, et al. (2008) A Transcriptome Database for Astrocytes, Neurons, and Oligodendrocytes: A New Resource for Understanding Brain Development and Function. J Neurosci 28: 264-278.

20. Makar TK, Bever CT, Singh IS, Royal W, Sahu SN, et al. (2009) Brain-derived neurotrophic factor gene delivery in an animal model of multiple sclerosis using bone marrow stem cells as a vehicle. J Neuroimmunol 210: 40-51. 